\title{
No Impact of Cerebellar Anodal Transcranial Direct Current Stimulation at Three Different Timings on Motor Learning in a Sequential Finger-Tapping Task
}

\author{
Carine Nguemeni*, Annika Stiehl ${ }^{\dagger}$, Shawn Hiew ${ }^{\dagger}$ and Daniel Zeller \\ Department of Neurology, University Hospital of Würzburg, Würzburg, Germany
}

Background: Recently, attention has grown toward cerebellar neuromodulation in motor

OPEN ACCESS

Edited by:

Stephane Perrey,

Université de Montpellier, France

Reviewed by: Anirban Dutta,

University at Buffalo, United States

Kazuhiro Sugawara,

Sapporo Medical University, Japan

Shapour Jaberzadeh,

Monash University, Australia

Aguida Soares Foerster,

Physiotherapie Bossert \&

Junggebauer, Germany

${ }^{*}$ Correspondence:

Carine Nguemeni

nguemeni_c@ukw.de

tThese authors have contributed equally to this work

Specialty section: This article was submitted to Brain Imaging and Stimulation, a section of the journal

Frontiers in Human Neuroscience

Received: 20 November 2020 Accepted: 13 January 2021

Published: 05 February 2021

Citation:

Nguemeni C, Stiehl A, Hiew S and Zeller D (2021) No Impact of Cerebellar Anodal Transcranial Direct Current Stimulation at Three Different

Timings on Motor Learning in a

Sequential Finger-Tapping Task. Front. Hum. Neurosci. 15:631517. doi: 10.3389/fnhum.2021.631517 learning using transcranial direct current stimulation (tDCS). An important point of discussion regarding this modulation is the optimal timing of tDCS, as this parameter could significantly influence the outcome. Hence, this study aimed to investigate the effects of the timing of cerebellar anodal tDCS (ca-tDCS) on motor learning using a sequential finger-tapping task (FTT).

Methods: One hundred and twenty two healthy young, right-handed subjects (96 females) were randomized into four groups (Duringsham, Before, Duringreal, After). They performed 2 days of FTT with their non-dominant hand on a custom keyboard. The task consisted of $40 \mathrm{~s}$ of typing followed by $20 \mathrm{~s}$ rest. Each participant received ca-tDCS (2 mA, sponge electrodes of $25 \mathrm{~cm}^{2}, 20 \mathrm{~min}$ ) at the appropriate timing and performed 20 trials on the first day (T1, $20 \mathrm{~min})$. On the following day, only 10 trials of FTT were performed without tDCS (T2, $10 \mathrm{~min}$ ). Motor skill performance and retention were assessed.

Results: All participants showed a time-dependent increase in learning. Motor performance was not different between groups at the end of T1 $(p=0.59)$. ca-tDCS did not facilitate the retention of the motor skill in the FTT at T2 $(p=0.27)$. Thus, our findings indicate an absence of the effect of ca-tDCS on motor performance or retention of the FTT independently from the timing of stimulation.

Conclusion: The present results suggest that the outcome of ca-tDCS is highly dependent on the task and stimulation parameters. Future studies need to establish a clear basis for the successful and reproducible clinical application of ca-tDCS.

Keywords: cerebellar tDCS, finger-tapping task, timing, motor learning, task retention

\section{INTRODUCTION}

Transcranial direct current stimulation (tDCS) is a non-invasive brain stimulation technique that has impressively grown in popularity in clinical research within the last two decades (Stagg et al., 2018). It has been widely used as a simple and safe method to modulate cortical excitability of the human brain both in the healthy 
and in the pathological context (Dedoncker et al., 2016; Angius et al., 2019; de Moura et al., 2019).

While most studies focus on the influence of tDCS on cortical areas, the interest in cerebellar transcranial direct current stimulation (ctDCS) has grown rather recently, mainly after Galea et al. (2009) described polarity-depended excitability changes using ctDCS. Twenty-five minutes of anodal stimulation increased the connectivity between the cerebellum and the primary motor cortex (M1), whereas connectivity was decreased by cathodal stimulation (Galea et al., 2009). An additional number of recent studies have shown that tDCS induces significant changes in cerebellar excitability (Ferrucci et al., 2015).

The cerebellum plays a critical role in motor learning, movement coordination, motor adaptation, and cognitive processing (Thach, 1998; Morton and Bastian, 2006; Buckner, 2013). Indeed, the connections between the cerebellum and the motor cortex are essential for the performance of daily life activities. This is because the cerebellum is closely connected to the motor cortex via multiple closed-loop circuits which optimize motor control and motor learning by refining motor inhibition (Wolpert et al., 2011). This represents a key feature of cerebrocerebellar interactions (Kelly and Strick, 2003). In this sense, cerebellar stimulation may functionally affect cerebrocerebellar interactions and modulate functions residing in the motor cortex and the whole brain (Priori et al., 2014). This also explains why any perturbation of the integrity of these interactions results in motor disorders including ataxia, dysmetria, dystonia (Manto et al., 2013).

Therefore, developing non-invasive strategies to modulate cerebellar excitability constitutes an interesting opportunity to further understand the cerebellar function and the potential benefit of cerebellar stimulation for patients with neurological disorders. Regarding motor learning, future applications could include improvement of fine motor functions (Priori et al., 2014) or improving balance control (Manto et al., 2013; Poortvliet et al., 2018), e.g., in stroke patients (Wiestler et al., 2011).

There are, however, several challenges that need to be overcome to use ctDCS successfully in research as well as in clinical settings. The human cerebellar cortex presents a complex architecture in a sheet-like structure and accordion-like folds containing layers of excitatory and inhibitory cells (Motolese et al., 2013). Applying stimulation in a precise direction parallel to the somatodendritic axis of the target cells, which was shown to generate the maximum effect on polarization (Bikson et al., 2004), is therefore not easy to accomplish. Also, inter-individual differences regarding skull thickness and cerebellar architecture limit the finding of congruent effects (Motolese et al., 2013; Guell and Schmahmann, 2020); even when study designs and paradigms are very similar (Rahman et al., 2014; Jalali et al., 2017). Thus, the results obtained from tDCS applied to the cerebrum cannot be simply transferred to the cerebellum (Motolese et al., 2013; van Dun et al., 2016). Furthermore, there is a critical lack of consensus about the application of tDCS (Rezaee and Dutta, 2019).
Therefore, a systematic investigation of the effects of different ctDCS protocols is needed (Ferrucci et al., 2015). This will help to generate maximum effects in further studies and to make ctDCS studies more comparable. Regarding ctDCS protocols, the effects of five main factors need to be investigated: the duration of stimulation, the current intensity, electrode size and placement, the number of ctDCS sessions, and the timing of stimulation (Stagg et al., 2011). In the present study, we focused on the timing of stimulation.

When referring to stimulation timing, one usually distinguishes between offline stimulation and online stimulation. Whereas in online stimulation participants perform a task during the stimulation, offline stimulation can be administered either before or after training a task. Many studies use one of the timing options without justifying their choice in detail (Thair et al., 2017). Not rarely, online stimulation is preferred and justified by simple reasons including: (1) a tight schedule; or (2) the assumption that both online and offline protocols induce the same polarity-specific effects (Schlerf et al., 2015). However, the timing of stimulation concerning the execution of a specific behavioral task might play a pivotal role in the overall outcome of tDCS. In opposition to reports from the stimulation of the motor cortex (Sriraman et al., 2014; Stagg et al., 2018), no study has yet investigated the effect of ctDCS timing on motor performance and retention in motor learning, comparing offline and online stimulation within the same study. Here, we sought to explore the impact of anodal ctDCS at different times (before, during, or after a task) on motor performance and retention using a sequential finger-tapping task (FTT). We hypothesized that anodal ctDCS applied during the task will improve task performance while anodal ctDCS applied after the task will improve consolidation and retention when compared to sham tDCS. A previous study demonstrated that a single session of $\mathrm{tDCS}$ to the prefrontal cortex before an implicit sequence learning task does not affect the performance on the task (Savic et al., 2017). As such, we also hypothesized that anodal ctDCS applied before the FTT will neither influence motor performance nor consolidation and will produce results similar to sham $\mathrm{tDCS}$.

The FTT represents a widely used procedural memory task to assess motor skill learning (Walker et al., 2002; Rasch et al., 2009). The cerebellum has been shown to play an important role in the correct execution of this type of motor sequence learning task (Penhune and Steele, 2012; Shimizu et al., 2017). Moreover, patients with cerebellar degeneration fail to show improvement in procedural learning (Pascual-Leone et al., 1993; Gómez-Beldarrain et al., 1998; Shin and Ivry, 2003).

Regarding the ideal cerebellar region to stimulate when modulating FTT performance, studies have analyzed the cerebellar-motor cortex connectivity governing upper-limb motor skill. The individual finger-specific activation shows only weak somatotopic organization with ipsilateral patterns of activation in the lobule V and VIII of the cerebellar hemisphere (Wiestler et al., 2011). Upper-limb representation is also more widely distributed touching face and mouth representation in the superior posterior cerebellum (lobule VI) and lower limb representations in the inferior posterior cerebellum (lobules 
VIIb-IX; Motolese et al., 2013). Data-driven from cerebellar functional magnetic resonance imaging indicated that the lobules I-VI and lobule VIII of the cerebellar hemisphere are activated during motor processing like moving a finger (Guell and Schmahmann, 2020). Altogether, these data hint at the stimulation of the posterior cerebellum as a good target to evaluate ctDCS on FTT. We chose this region as our target using an electrode montage (Galea et al., 2009) which can effectively affect the lobules VIIb, VIII, and IX in the ipsilateral cerebellum (Rezaee and Dutta, 2019).

Here, we specifically focused our evaluation on the timing of anodal stimulation in one session because multiple studies have suggested a facilitatory effect of the anodal polarity on motor learning with ctDCS (Galea et al., 2011; Jayaram et al., 2012; Hardwick and Celnik, 2014; Herzfeld et al., 2014; Priori et al., 2014; Cantarero et al., 2015). More, a single stimulation of the cerebellum has been shown to lead to significant retention of fine motor skills (Wessel et al., 2016).

\section{MATERIALS AND METHODS}

\section{Participants}

One hundred and twenty two healthy, right-handed young adults (96 females, 26 males, age range: 19-27 years, mean age: $22.5 \pm 2.2$ ) were recruited for this study. All participants were university students sharing a comparable level of education. Before recruitment, the participants received an e-mail detailing the experimental timeline, the inclusion and exclusion criteria, and the questionnaires. The Oldfield questionnaire (Oldfield, 1971) was used to determine handedness. The Pittsburgh Sleep Quality Index (PSQI) questionnaire (Buysse et al., 1989) was used to evaluate the sleep quality of the subjects. We also evaluated the subjective self-estimated hours of sleep per night. Finger dexterity was reported as the usual number of fingers of the non-dominant hand (left-hand for all participants) used when typing on a computer keyboard. Exclusion criteria were: left-handedness, neurological or psychiatric disorders (in particular, history of depression, epilepsy, brain injury, dizziness, and vertigo), severe sleep disorders (PSQI $\geq 8$ ), medication or substances affecting the central nervous system, having undergone brain or vertebral column surgery, presence of medical devices (surgical clips, cochlear implants, drug pumps, pacemakers, et cetera), diagnosis of learning difficulties (dyslexia, dyscalculia, language impairments), tattoos or piercings on the scalp, professional level of typing or piano playing, pregnancy. Upon completion of all sessions, the participants received $8 €$ per hour as compensation. The data of four participants were excluded due to the automatic execution of a wrong sequence by the program during the task. Another six were excluded because they had consumed cannabis or alcohol, practiced the sequence or slept less than $4 \mathrm{~h}$ between the first and the second day of experiment. Thus, a total of 28 participants per group were included in the analysis.

The study conformed to the principles of the declaration of Helsinki and was approved by the local ethics committee of the Medical Faculty at the University of Würzburg. All subjects gave their written informed consent before the investigation. All participants were naive to the rationale of the experiment.

\section{Research Design}

The participants were tested in a double-blind between-subject design as follows: (1) the group "Before" received cerebellar anodal tDCS (ca-tDCS) before the motor learning task; (2) the group "During sham" received sham tDCS during the task; (3) the group "During ${ }_{\text {real }}$ " had real ca-tDCS during the task; and (4) the participants in the "After" group were stimulated with ca-tDCS after the task. The experiments were conducted on two consecutive days with $24 \mathrm{~h}$ between sessions. On the first day, subjects received tDCS and performed the motor learning task. On the second day, participants reported on their current health condition, their caffeine and drug consumption, their medication intake as well as the number of hours of sleep in the night between sessions. They subsequently performed the motor task. All experiments were conducted in the same room by the same investigator between 7 a.m. and 1:15 p.m.

\section{Finger Tapping Task (FTT)}

Figure 1 provides a schematic view of the experiment. We used a computerized version of the sequential FTT initially developed by Karni et al. (1995). The study was designed using OpenSesame_3.1.4-wind32 (RRID:SCR_002849, Mathôt et al., 2012). Four keys, located in ergonomic positions on a computer keyboard were used (with keys-to-number assignment: 1 = index finger [ $\mathrm{N}$ key], 2 = middle finger [G key], 3 = ring finger [D key], $4=$ little finger [Y key]). The corresponding sequence was "Y-ND-G-Y” on a keyboard with a standard QWERTZ layout (Fujitsu Technology Solutions). To limit the errors, white squared paper straps with the black written numbers $(1,2,3$, and 4) were taped over the keys, and all the letter keys that were not required for the task were removed from the keyboard. The task required to repeat, as quickly and accurately as possible, a sequence of five finger-movements (4-1-3-2-4) using the left, non-dominant hand for a period of $40 \mathrm{~s}$ followed by a break of $20 \mathrm{~s}$. During the $20 \mathrm{~s}$ of the break, the message " $20 \mathrm{~s}$ break" was displayed on the screen. A block lasted 1-min corresponding to $40 \mathrm{~s}$ of test and $20 \mathrm{~s}$ of the break. On the first day, participants were familiarized with the sequence during the one familiarization block before starting the real test. The familiarization trial did not differ from the other blocks and there was no notification between the familiarization trial and the beginning of the real session. The training session consisted of 20 blocks and lasted $20 \mathrm{~min}$. All participants were instructed to concentrate on the task and not to talk. At the end of the session, participants were instructed not to practice the sequence outside of the experiment and to keep their usual sleep schedule between the 2 days of the experiment. On the second day, there was no initial familiarization trial and the subjects performed only 10 blocks of the same finger tapping sequence.

\section{Cerebellar Anodal tDCS (ca-tDCS)}

Anodal cerebellar tDCS (DC-Stimulator Plus, neuroConn, Ilmenau, Germany, RRID:SCR_015520) was delivered through two $5 \mathrm{~cm} \times 5 \mathrm{~cm}\left(25 \mathrm{~cm}^{2}\right)$ electrodes soaked in a $0.9 \%$ 


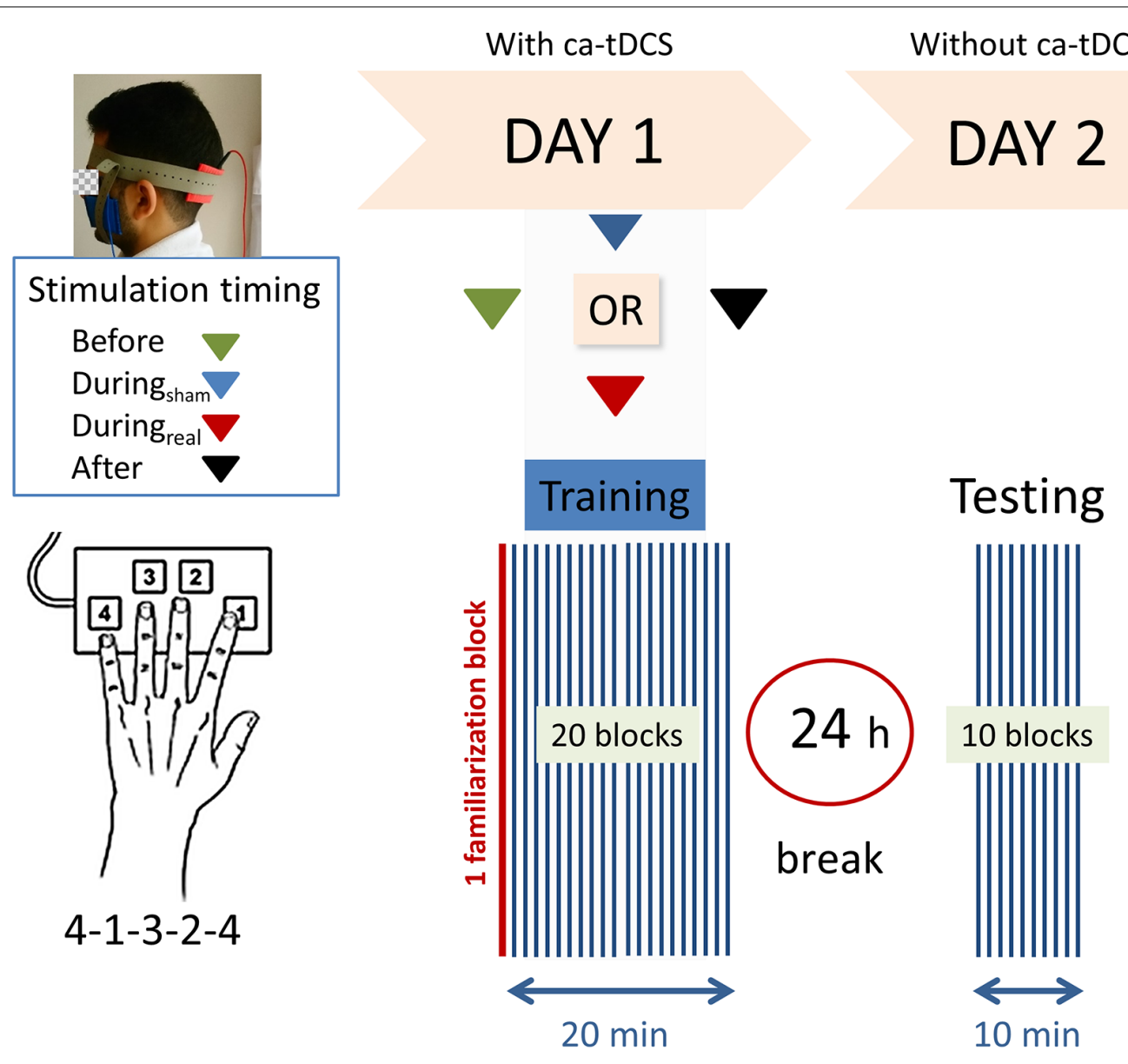

FIGURE 1 | Experimental design. Right-handed participants were asked to perform as rapidly and accurately as possible a 5-digits sequence (4-1-3-2-4) of a finger-tapping task (FIT) on a modified keyboard using their left hand. One block consisted of $40 \mathrm{~s}$ of tapping and $20 \mathrm{~s}$ of rest. After a familiarization block (marked in red), the subjects performed 20 blocks on the first training day. Following a $24 \mathrm{~h}$ break, a recall session consisting of 10 blocks was completed. Anodal transcranial direct current stimulation was applied to the ipsilateral cerebellar hemisphere on day 1 with the reference electrode on the buccinator muscle at the following timings with respect to the training: (1) "Before," (2) "Duringsham," (3) "Duringreal," or (4) "After."

$\mathrm{NaCl}$-solution for at least $10 \mathrm{~min}$. The anodal electrode was placed over the left cerebellar cortex, $3 \mathrm{~cm}$ lateral to the inion, and the cathodal electrode was placed on the left buccinator muscle (Galea et al., 2011). For the "During" groups, both the participants and the experimenter were blinded to the type of stimulation (sham or anodal) using the "study mode" implemented in the tDCS device. At the onset of stimulation, the current was increased in a ramp-like fashion for $10 \mathrm{~s}$. For real stimulation, a $2 \mathrm{~mA}$ current (current density $0.08 \mathrm{~A} / \mathrm{cm}^{2}$ ) was applied for $20 \mathrm{~min}$. For sham stimulation, tDCS started with a short linear fade-in phase, followed by $2 \mathrm{~mA}$ of direct current for $30 \mathrm{~s}$ and a short linear fade-out phase. During the main time of the stimulation, only small current pulses occurred every $550 \mathrm{~ms}(110 \mu \mathrm{A}$ over $15 \mathrm{~ms}$ ) to check the impedance. The total stimulation session lasted $20 \mathrm{~min}$, independently of the stimulation mode (real or sham). The timing of stimulation relative to the FTT (before, during, or after) varied according to the experimental group. All participants were instructed not to talk during stimulation. tDCS was well tolerated and there were no adverse effects.

\section{Data Analysis and Statistics}

To evaluate FTT performance, we calculated the number of correct sequences per block on the first and second sessions for each participant. The change in performance throughout the training session on the first day was considered a measure of online learning. The online gains were calculated by subtracting the average performance in the first three blocks (PRE) from the average performance in the last three blocks (POST) on the first day of training:

$$
\text { Online gains }=P O S T-P R E
$$

The difference between the average performance in the last three blocks of learning (POST) and the first three blocks of the recall session (RT) represented an index of off-line consolidation (Saucedo Marquez et al., 2013):

$$
\text { Offline gains }=R T-\text { POST }
$$


SPSS version 22 (IBM, RRID:SCR_002865) was used for inferential analysis. The Shapiro-Wilk test was used to test normality and the Levene test was used to test homogeneity of variance. The Kruskal-Wallis one-way analysis of variance was used in case of non-normal distribution. We compared the normally distributed data using repeated-measures ANOVA with GROUP as between subjects' factor ("Before," "During sham," "During $_{\text {real }}$," and "After") and TIME as a withinsubjects factor (T1 and T2). Online and offline gains for each group were compared using the one-way ANOVA test. The effect sizes were calculated as partial $\eta^{2}$. Mauchly's test was used to test the assumption of sphericity between the conditions and Greenhouse-Geisser correction was applied in case of violation. Bonferroni correction was applied for multiple comparisons. Data are reported as mean \pm standard error of the mean. The significance level was set at $p<0.05$.

\section{RESULTS}

\section{Demographic and Psychometric Data}

Demographic and psychometric data are summarized in Table 1. Participants were between 19 and 27 years old, with no significant age differences between groups. All subjects were right hand dominant (handedness score 20-100\%). There were no significant differences between the groups regarding sleep quality (PSQI) within the last 4 weeks before the experiment, self-estimated hours of sleep per night, and finger dexterity.

\section{Effect of Cerebellar Anodal-tDCS on FTT Performance}

Subjects received the tDCS stimulation at different timings (before, during, or after the task). No adverse effect was reported during or following the stimulation. On day 1, participants increased the number of correct sequences during the task as shown by the increasing learning curve across blocks (Figure 2A). We performed a two-way repeated-measures ANOVA with GROUP as between subjects' factor and TIME as a within-subjects' factor. There was no significant GROUP $\times$ TIME interaction on day $1\left(F_{(25.78,902.43)}=0.82, p=0.72, \eta^{2}=0.023\right)$ indicating that the average number of correct sequences per block was comparable between groups and that the timing of tDCS did not affect that performance. We found a main effect of time $\left(F_{(8.59,902.43)}=60.53, p<0.001, \eta^{2}=0.36\right)$ indicating significant online learning (practice effect) in every group.

Similarly, there was no significant GROUP $\times$ TIME interaction on day 2 ( $\left.F_{(12.74,445.74)}, p=0.091, \eta^{2}=0.043\right)$. Similarly to day 1 , we found a significant main effect of time $\left(F_{(4.25,445.74)}=1.57, p=0.03, \eta^{2}=0.04\right)$ underlining that the average number of correct sequences performed per block further improved on the second day of FTT.

One-way ANOVA was used to compare the overall average of correct sequences for 20 blocks and 10 blocks on day 1 and day 2, respectively, in each group. We found no between-group differences on the overall average of correct sequences per group on day $1\left(F_{(0.96,52)}, p=0.42, \eta^{2}=0.12\right.$, Figure $\left.2 B\right)$. Similarly, there was no significant difference on the overall average of correct sequences per group on day $2\left(F_{(39.2,431)}, p=0.91, \eta^{2}=0.04\right.$, Figure 2C).

\section{Effects of the Timing of Cerebellar Anodal tDCS on Online and Offline Learning}

The results of the one-way ANOVA are shown in Table 2. We evaluated the online gain in the performance by subtracting the mean of correct sequences in the first three blocks from the mean of correct sequences in the last three blocks for each group and day. There was no significant difference between groups at day $1(p=0.59$, Figure 3A). To evaluate the consolidation/retention effect, we subtracted the average of the correct sequences in the first three blocks of the second day from the last three blocks in

TABLE 1 | Summary of demographic and psychometric parameters.

\begin{tabular}{|c|c|c|c|c|c|}
\hline & Groups & $N$ & Mean of ranks & $x^{2}$ & $p$ \\
\hline & Before & 28 & 57.30 & & \\
\hline & Duringreal $_{\text {r }}$ & 28 & 58.09 & 0.44 & 0.93 \\
\hline \multirow[t]{3}{*}{ Handedness } & During sham & 28 & 51.61 & & \\
\hline & Before & 28 & 53.18 & & \\
\hline & Duringreal & 28 & 59.54 & 1.94 & 0.59 \\
\hline & Before & 28 & 51.46 & & \\
\hline & Duringreal & 28 & 61.70 & 2.3 & 0.51 \\
\hline & After & 28 & 60.39 & & \\
\hline \multirow[t]{3}{*}{ Self-estimated sleep/night (hours) } & During $_{\text {sham }}$ & 28 & 56.61 & & \\
\hline & Before & 28 & 56.34 & 0.19 & 0.98 \\
\hline & Duringreal & 28 & 58.38 & & \\
\hline Total of participants & $N=$ & 112 & & & \\
\hline
\end{tabular}



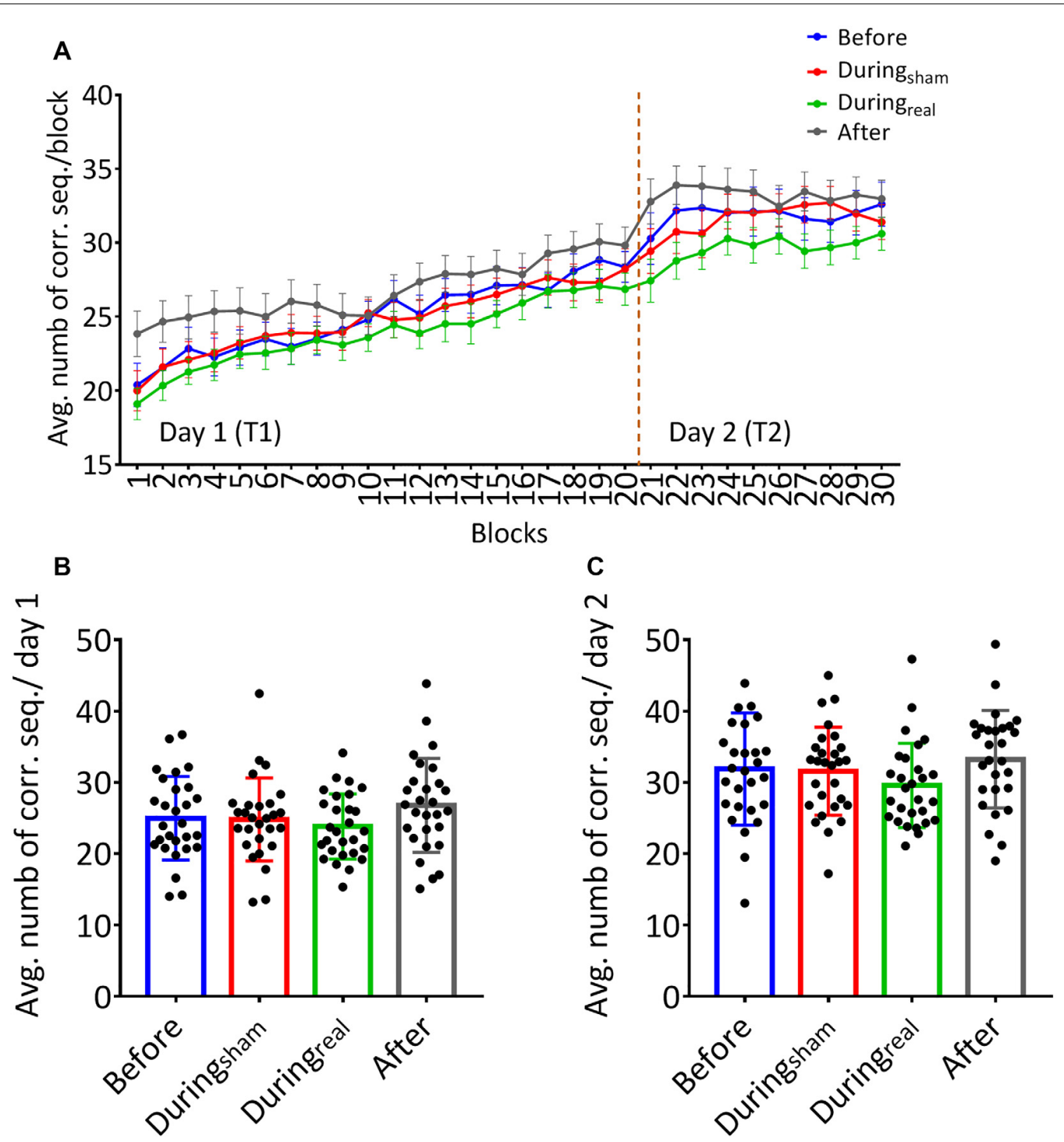

FIGURE 2 | (A) Learning curves of the finger sequence tapping task. The number of correct sequences tapped during each block was collected and the average per block was calculated for each group on day 1 and day 2 . Solid lines indicate group means, error bars indicate standard error of means (sem, $n=28$ per group). (B) Mean performance on day 1. The overall average of correct sequences was calculated for the 20 blocks completed on day 1 for each group. (C) Mean performance on day 2. The overall average of correct sequences was calculated across the 10 blocks performed on day 2 for each group (mean performance \pm SD, $n=28$ per group).

the first day. Again, there was no significant difference between groups $(p=0.27$, Figure 3B).

\section{DISCUSSION}

We evaluated the impact of timing of ca-tDCS applied before, during, or after an FTT (relative to sham) on the online motor performance and task retention. We found that all participants showed an improvement in performance as measured by the number of correct FTT sequences for each session. There was no effect of cerebellar tDCS on motor learning. Participants who received sham stimulation produced the same average of correct responses as participants who received active anodal
tDCS either before, during, or after the task. There were no baseline differences in motor performance at the beginning of the task on day 1. Similarly, there was no difference in online gains or offline improvement between groups. Overall, our results underline a lack of effect of ca-tDCS on motor learning in the context of FTT.

\section{Cerebellar tDCS Did Not Affect Online Learning}

In the context of this study, it is important to discriminate between motor adaptation and skill learning. While in motor adaptation, the learner adapts to the error induced by a perturbation and shows an aftereffect when the perturbation 
TABLE 2 | Online and offline gains in the finger-tapping task (FIT)

\begin{tabular}{|c|c|c|c|c|c|c|c|}
\hline & & $N$ & Mean & Standard deviation & $\boldsymbol{F}$ & Partial $\eta^{2}$ & $p$ \\
\hline Online & During $_{\text {sham }}$ & 28 & 6.38 & 3.73 & & & \\
\hline gains & Before & 28 & 6.82 & 4.55 & & & \\
\hline \multirow[t]{2}{*}{ day 1} & Duringreal & 28 & 6.65 & 5.61 & 0.65 & 0.01 & 0.59 \\
\hline & After & 28 & 5.32 & 3.47 & & & \\
\hline Offline & During $_{\text {sham }}$ & 28 & 2.64 & 3.42 & & & \\
\hline \multirow[t]{3}{*}{ gains } & Before & 28 & 3.18 & 4.32 & & & \\
\hline & Duringreal & 28 & 1.61 & 4.21 & 1.34 & 0.03 & 0.27 \\
\hline & After & 28 & 3.68 & 4.19 & & & \\
\hline Total of participants & $N=$ & 112 & & & & & \\
\hline
\end{tabular}
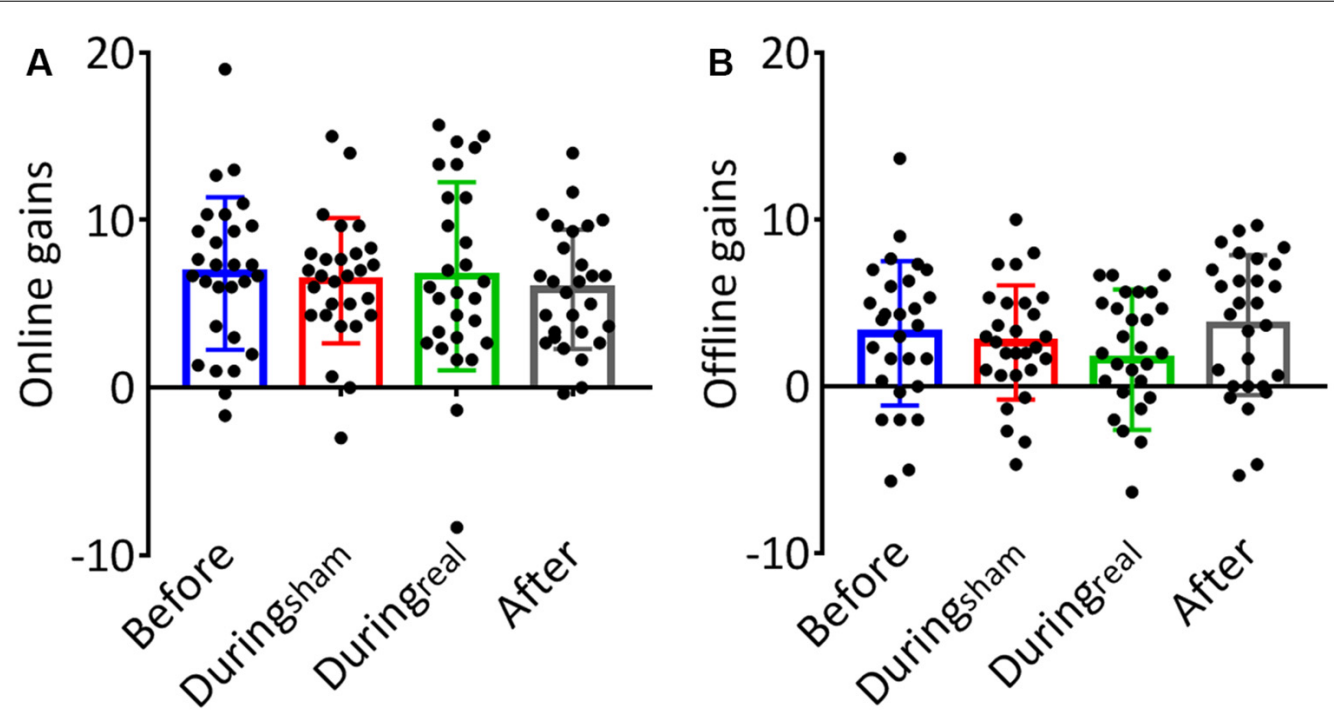

FIGURE 3 | (A) Online gains. The change in performance throughout the training session on day 1 was considered a measure of online (or practice-dependent) learning. This gain was calculated by subtracting the average number of correct sequences in the first three blocks from the average number of correct sequences in the last three blocks on day 1. (B) Offline gains. The change in performance from the last three blocks of learning (day 1 ) to the first three blocks in the recall session (day 2) represented an index of offline gains. These gains were considered a measure of offline memory consolidation for each group (mean gain \pm SD, $n=28$ per group).

is removed (Martin et al., 1996), motor skill learning is evaluated through exposure to a novel motor task. Successful learning is measured through the reduction of errors and performance improvement beyond baseline levels (Reis et al., 2009). To our knowledge, our study is the first to evaluate the impact of timing of a single ca-tDCS on the performance in an FTT, a motor sequence learning task that has been commonly associated with cerebellar activation (Witt et al., 2008). While cerebellar activity has traditionally been associated with externally cued movements, it is also observed in motor tasks driven by internal cues (Grafton et al., 1992). Specifically, lobule VIII of the cerebellum is recruited during out-of-phase movements related to the motor coordination of finger movements (Habas et al., 2004). Moreover, righthanded finger-tapping activated right cerebellar lobules IV-V and VIII (Stoodley et al., 2012). In a recent modeling study, the electrode montage used in our current study has consistently been demonstrated to generate an electrical field with its peak strength within cerebellar lobules VII and VIII (Moussa-Tooks et al., 2020).
The participants in this study did not show baseline differences in task performance. Average age, sleep quality, handedness, and finger dexterity did not differ between the four groups. We evaluated and compared the change in motor performance during the task as a measure of online gains and found no differences between the groups. Our result underlines a lack of effect which is in contradiction with some previous studies of others showing a positive effect of ca-tDCS on motor learning in various tasks. For example, ca-tDCS enhanced motor performance by improving the rate of adaptation when compared to sham stimulation (Jayaram et al., 2011). In paradigms that specifically involve the upper limb, ca-tDCS improved the accuracy index in a skilled angle tracking task (Shah et al., 2013) and reduced the response time and the number of errors in the serial response time task (Ehsani et al., 2016; Samaei et al., 2017). Controversially, other authors reported a lack of effects of the anodal stimulation in a specific upper limb test with an adaptation component like the visuomotor adaptation task (Panouillères et al., 2015; Liew et al., 2018). Moreover, a study from Taubert et al. (2016) suggested 
that ca-tDCS impaired motor memory acquisition and increased motor errors during the re-acquisition of an original motor memory in a force field perturbation-reaching task.

In most of the above-mentioned studies, the current was delivered during the task, ipsilateral to the trained $\operatorname{limb}$ at a current density of $0.08 \mathrm{~mA} / \mathrm{cm}^{2}$. This condition is comparable to the "Duringreal" group in our study where participants received the stimulation during the task. The "During no significant online gains compared to the "During sham" group that received sham stimulation. We also evaluated the online gains when the active stimulation was delivered before or after the task and found similar results, which confirmed the lack of effect of ca-tDCS.

It is however important to mention that we specifically stimulated the left cerebellum, whereas most of the up-todate studies published have stimulated the right cerebellum. This difference in the hemisphere stimulation might influence the cortical response (Schlerf et al., 2015) indicating some laterality differences in the cerebellar-motor cortex connectivity. As such, they observed in right-handed individuals that the connection between the right cerebellum and left M1 was stronger than the contralateral network. This possibility of lateralization might have influenced the results in our right-handed participants.

\section{Cerebellar tDCS Did Not Affect Task Retention (Offline Gains)}

Motor performance in the FTT was re-assessed after a break of $24 \mathrm{~h}$ as a measure of task retention and offline learning. Our results indicated no impact of tDCS on task retention. Similarly as discussed for the online gains, reports about the effects of cerebellar anodal tDCS on offline gains are controversial. For instance, Taubert et al. (2016) reported an impaired early adaptation in a force field perturbation task at $24 \mathrm{~h}$ post-tDCS. This negative effect was also found as a decrease of reaction time in a serial reaction time task following anodal cerebellar stimulation. However, two other studies have reported a positive outcome on offline learning following ca-tDCS in the above-mentioned serial reaction task. This was reflected by a greater reduction in response time, but not the number of errors, in individuals over 40 years (Samaei et al., 2017) and a greater reduction in the number of errors and faster response time in subjects younger than 40 years old (Ehsani et al., 2016). In all those studies the stimulation was applied during the task in a single session. Similar to the result found with the "Before" group of our study, Foerster et al. (2017) have reported the absence of effect of ca-tDCS on offline learning in an adaptive balance control task when the stimulation was applied before the task. In this study, we have also tested a condition where anodal stimulation was applied after the task ("After" group) as another possible offline stimulation design. We found no difference between the groups that received the tDCS before or after completing the task on day 2. This result shows that tDCS after the task did not affect retention. From the literature, it is unclear whether cerebellar tDCS has any effect on motor learning, performance, and retention when applied during or after the task. Our current results point toward a lack of effect in both timings.

\section{Inconsistency in tDCS Studies and Limitations}

Recent studies have called attention to several aspects of the methodology for tDCS (Thair et al., 2017). One of them includes the variability in performance level that was not assessed in this study. tDCS effects could have been masked by large interindividual variability in motor skills. Another important factor is the hair thickness and texture that can increase impedance and thus allow less current to reach brain tissue (Doyon and Benali, 2005). The impedance in this study was maintained below $15 \mathrm{~K} \Omega$ throughout the stimulation session. However, we did neither protocol individual impedance in detail nor did we map patients' skulls or brain tissue and thus, we do not know whether groups differed in these matters significantly.

A potential contributor to the null effect might be the specificity of the electrode positioning that has been shown effective to facilitate motor adaptation (Morton and Bastian, 2006; Jayaram et al., 2012; Poortvliet et al., 2018). Ability to adapt to an external output and ability to learn a sequence of the movement have been often treated as distinct forms of motor learning. Both forms of motor learning might be supported by distinct neural substrates. Thus, work by Imamizu et al. (2000) has consistently shown brain activation in the cerebellar regions surrounding the posterior superior fissure of the cerebellum during adaptation of movements to differing visual distortions. On the other hand, cortico-cerebellar anatomical systems are crucial for mediating the acquisition and the execution of motor skills (Hikosaka et al., 2002; Doyon and Benali, 2005). These aspects of learning are not necessarily dissociated from real behaviors. Therefore, we argue that the stimulation of the posterior part of the cerebellum could modify performance in skill learning as underlined by the work of others (Wessel et al., 2016). However, it is now imperative to consider the use of additional techniques to the protocol that may map the cerebellum and its surrounding skull as well as the functional areas being investigated. Such techniques include the use of computational models, MEP or TMS (Furuya et al., 2014; Fan et al., 2017). Cerebellar Lobules Optimal Stimulation (CLOS) is an example of an open-source computational modeling pipeline that could support a critical and rational design as well as optimization of neuromodulation (Rauscher et al., 2020). The implementation of those tools represents an important change of the practice in the field that will improve the reproducibility of the studies.

The blinding methods have also been questioned in recent studies. The design of sham stimulation was conducted similarly to other studies (e.g., Jayaram et al., 2012; Manto et al., 2013; Poortvliet et al., 2018). Gandiga et al. (2006) have suggested that good blinding effects are achieved when using a ramp-like fade-in phase of approximately $10 \mathrm{~s}$ and turning off the current after $30 \mathrm{~s}$ in sham stimulation in comparison to a real stimulation applied at $1 \mathrm{~mA}$ for $20 \mathrm{~min}$. These findings have been implemented by multiple tDCS studies presuming good blinding with similar protocols (Fregni et al., 2005, 
2006). Based on the evaluation that we performed in half of our participants, only two subjects that received the sham stimulation were able to appropriately identify it. This means that most of the sham-stimulated participants thought that they have received an active stimulation. This result has been added to the Supplementary Material. However, we aim to monitor and assess the influence of the subjective experience of sensations during the stimulation as well as subjective allocation to either sham or anodal group to control insufficient blinding in the future.

The population in the present study was young, healthy, and well-educated. Participants performed already with very high accuracy in the first block and could therefore not improve much further during the experiments. Indeed, Furuya et al. (2014) compared the effects of tDCS on the fine control of sequential finger movements in highly trained pianists and musically untrained individuals. They demonstrated an improvement of fine motor control in both hands in musically untrained controls, but the deterioration in pianists following anodal tDCS over the contralateral cortex. This underlines that the effects of tDCS might be expertise-dependent. In the current study, participants were naïve to the task. We also purposely excluded subjects with extended typing experience, including piano playing. We can therefore assume that the subjects had a similar level of initial performance. This was also confirmed by the absence of difference in correct response in the first block of T1. We could speculate the real statistical effect hidden by the very high initial performance would be uncovered in older participants or participants with cerebellar functional impairment. However, a recent study has also shown a lack of cerebellar tDCS effect on learning of a complex whole dynamic balance test in middle-age adults (Rauscher et al., 2020) underlining that parameters beyond the age of participants are responsible for the null effect obtained in our study.

It is important to note that the results presented here and their interpretation are limited to the effect of a single stimulation. Possibly, multiple sessions could have yielded a different result. Anodal transcranial stimulation of the primary motor cortex over five consecutive days has been shown to enhance motor performance in a grip task in healthy young subjects (Fan et al., 2017). Cerebellar anodal tDCS over 3 days improved the online performance of healthy participants in a sequential visual isometric punch task (Cantarero et al., 2015). Therefore, we cannot exclude that with only one tDCS session, the effects may have been minimal, whereas multiple sessions could have shown larger and longer-lasting effects. However, there is no clear evidence to strongly support the effectiveness of multiple sessions over a single session when it comes to cerebellar stimulation. A recent systematic review summarizing the effects of cerebellar tDCS on motor learning has concluded to a potential positive effect of a single session of ca-tDCS in improving short to long-term motor skill learning beyond the training period (Kumari et al., 2019).

Finally, we cannot exclude that the effect of cerebellar at-DCS might have been missed because of the sample size
( $N=28$ per group). However, based on previous comparable studies performed with a similar number of subjects per group, we had performed an a-priori power analysis and had found that 49 subjects in total would be required to achieve a power of 0.8 . A total of 112 participants were included in the current study, yet yielding a lack of effect. It is therefore possible that tDCS studies and especially cerebellar tDCS studies require substantially larger sample sizes because of the very small effects. To overcome the recruitment limitation, the pooling of data from several studies with small samples but similar experimental designs will create large data sets that might allow the estimation of efficacy much more precisely (Minarik et al., 2016).

\section{CONCLUSION AND IMPLICATIONS}

Cerebellar anodal t-DCS neither facilitated learning, nor retention in a FTT in young and healthy subjects. Several reasons explaining these results have been raised above. Our results call for a careful design of experiments using tDCS and underline the need for further investigation regarding the timing of its application, electrode placement, and intensity of the current as well as the choice of the target population in neurological studies. The individual differences, the sensitivity to tDCS, and the inter-population variability are among the parameters that could also play a critical role in the outcome of tDCS applications. It is becoming evident that heterogeneous and limited effects of tDCS including ours are calling for an urgent need to develop biomarkers that can help to predict individual response to non-invasive stimulation. We also need to define personalized targets and optimal parameters for the success of the intervention before moving to the clinical context where robust and consistent effects are expected despite the high variability of the subjects' condition.

\section{DATA AVAILABILITY STATEMENT}

The original contributions presented in the study are included in the article/Supplementary Material, further inquiries can be directed to the corresponding author.

\section{ETHICS STATEMENT}

The studies involving human participants were reviewed and approved by the ethics commission of the university hospital of Würzburg, Germany. The patients/participants provided their written informed consent to participate in this study.

\section{AUTHOR CONTRIBUTIONS}

$\mathrm{CN}$ : conceptualization, investigation, data acquisition, formal analysis, writing-original draft, writing-review and editing. AS and SH: recruitment, investigation, data acquisition, and formal analysis. DZ: conceptualization, writing-review and editing, funding acquisition, project administration, resources, 
and supervision. All authors contributed to the article and approved the submitted version.

\section{FUNDING}

The author(s) disclosed receipt of the following financial support for the research, authorship, and/or publication of this article: this work was supported by the German Foundation for Research; DFG project No. 286012902. CN is currently supported by the grant P1180017 from the Hertie Foundation. The funding sources have no involvement in the study design, collection,

\section{REFERENCES}

Angius, L., Santarnecchi, E., Pascual-Leone, A., and Marcora, S. M. (2019). Transcranial direct current stimulation over the left dorsolateral prefrontal cortex improves inhibitory control and endurance performance in healthy individuals. Neuroscience 419, 34-45. doi: 10.1016/j.neuroscience.2019. 08.052

Bikson, M., Inoue, M., Akiyama, H., Deans, J. K., Fox, J. E., Miyakawa, H., et al. (2004). Effects of uniform extracellular DC electric fields on excitability in rat hippocampal slices in vitro. J. Physiol. 557, 175-190. doi: 10.1113/jphysiol.2003. 055772

Buckner, R. L. (2013). The cerebellum and cognitive function: 25 years of insight from anatomy and neuroimaging. Neuron 80, 807-815. doi: 10.1016/j.neuron. 2013.10.044

Buysse, D. J., Reynolds, C. F. III., Monk, T. H., Berman, S. R., and Kupfer, D. J. (1989). The Pittsburgh Sleep Quality Index: a new instrument for psychiatric practice and research. Psychiatry Res. 28, 193-213. doi: 10.1016/01651781(89)90047-4

Cantarero, G., Spampinato, D., Reis, J., Ajagbe, L., Thompson, T., Kulkarni, K., et al. (2015). Cerebellar direct current stimulation enhances on-line motor skill acquisition through an effect on accuracy. J. Neurosci. 35, 3285-3290. doi: 10.1523/JNEUROSCI.2885-14.2015

de Moura, M., Hazime, F. A., Marotti Aparicio, L. V., Grecco, L. A. C., Brunoni, A. R., and Hasue, R. H. (2019). Effects of transcranial direct current stimulation (tDCS) on balance improvement: a systematic review and metaanalysis. Somatosens. Mot. Res. 36, 122-135. doi: 10.1080/08990220.2019. 1624517

Dedoncker, J., Brunoni, A. R., Baeken, C., and Vanderhasselt, M. A. (2016). A systematic review and meta-analysis of the effects of transcranial direct current stimulation (tDCS) over the dorsolateral prefrontal cortex in healthy and neuropsychiatric samples: influence of stimulation parameters. Brain Stimul. 9, 501-517. doi: 10.1016/j.brs.2016.04.006

Doyon, J., and Benali, H. (2005). Reorganization and plasticity in the adult brain during learning of motor skills. Curr. Opin. Neurobiol. 15, 161-167. doi: 10.1016/j.conb.2005.03.004

Ehsani, F., Bakhtiary, A. H., Jaberzadeh, S., Talimkhani, A., and Hajihasani, A. (2016). Differential effects of primary motor cortex and cerebellar transcranial direct current stimulation on motor learning in healthy individuals: a randomized double-blind sham-controlled study. Neurosci. Res. 112, 10-19. doi: 10.1016/j.neures.2016.06.003

Fan, J., Voisin, J., Milot, M. H., Higgins, J., and Boudrias, M. H. (2017). Transcranial direct current stimulation over multiple days enhances motor performance of a grip task. Ann. Phys. Rehabil. Med. 60, 329-333. doi: 10.1016/j.rehab.2017.07.001

Ferrucci, R., Cortese, F., and Priori, A. (2015). Cerebellar tDCS: how to do it. Cerebellum 14, 27-30. doi: 10.1007/s12311-014-0599-7

Foerster, A., Melo, L., Mello, M., Castro, R., Shirahige, L., Rocha, S., et al. (2017). Cerebellar transcranial direct current stimulation (ctDCS) impairs balance control in healthy individuals. Cerebellum 16, 872-875. doi: 10.1007/s12311017-0863-8

Fregni, F., Boggio, P. S., Nitsche, M., Bermpohl, F., Antal, A., Feredoes, E., et al. (2005). Anodal transcranial direct current stimulation of prefrontal cortex analysis of the data, their interpretation, the writing of the report as well as the decision to submit the article for publication.

\section{ACKNOWLEDGMENTS}

We thank the healthy participants for taking part in this study.

\section{SUPPLEMENTARY MATERIAL}

The Supplementary Material for this article can be found online at: https://www.frontiersin.org/articles/10.3389/fnhum. 2021.631517/full\#supplementary-material.

enhances working memory. Exp. Brain Res. 166, 23-30. doi: 10.1007/s00221005-2334-6

Fregni, F., Gimenes, R., Valle, A. C., Ferreira, M. J., Rocha, R. R., Natalle, L., et al. (2006). A randomized, sham-controlled, proof of principle study of transcranial direct current stimulation for the treatment of pain in fibromyalgia. Arthritis Rheum. 54, 3988-3998. doi: 10.1002/art.22195

Furuya, S., Klaus, M., Nitsche, M. A., Paulus, W., and Altenmuller, E. (2014). Ceiling effects prevent further improvement of transcranial stimulation in skilled musicians. J. Neurosci. 34, 13834-13839. doi: 10.1523/JNEUROSCI. 1170-14.2014

Galea, J. M., Jayaram, G., Ajagbe, L., and Celnik, P. (2009). Modulation of cerebellar excitability by polarity-specific noninvasive direct current stimulation. J. Neurosci. 29, 9115-9122. doi: 10.1523/JNEUROSCI.218409.2009

Galea, J. M., Vazquez, A., Pasricha, N., de Xivry, J. J., and Celnik, P. (2011). Dissociating the roles of the cerebellum and motor cortex during adaptive learning: the motor cortex retains what the cerebellum learns. Cereb. Cortex 21, 1761-1770. doi: 10.1093/cercor/bhq246

Gandiga, P. C., Hummel, F. C., and Cohen, L. G. (2006). Transcranial DC stimulation (tDCS): a tool for double-blind sham-controlled clinical studies in brain stimulation. Clin. Neurophysiol. 117, 845-850. doi: 10.1016/j.clinph.2005. 12.003

Gómez-Beldarrain, M., García-Moncí, J. C., Rubio, B., and Pascual-Leone, A. (1998). Effect of focal cerebellar lesions on procedural learning in the serial reaction time task. Exp. Brain Res. 120, 25-30. doi: 10.1007/s0022100 50374

Grafton, S. T., Mazziotta, J. C., Presty, S., Friston, K. J., Frackowiak, R. S., and Phelps, M. E. (1992). Functional anatomy of human procedural learning determined with regional cerebral blood flow and PET. J. Neurosci. 12, 2542-2548. doi: 10.1523/JNEUROSCI.12-07-02542.1992

Guell, X., and Schmahmann, J. (2020). Cerebellar functional anatomy: a didactic summary based on human fMRI evidence. Cerebellum 19, 1-5. doi: 10.1007/s12311-019-01083-9

Habas, C., Axelrad, H., and Cabanis, E.-A. (2004). The cerebellar second homunculus remains silent during passive bimanual movements. Neuroreport 15, 1571-1574. doi: 10.1097/01.wnr.0000133970.53139.e3

Hardwick, R. M., and Celnik, P. A. (2014). Cerebellar direct current stimulation enhances motor learning in older adults. Neurobiol. Aging 35, 2217-2221. doi: 10.1016/j.neurobiolaging.2014.03.030

Herzfeld, D. J., Pastor, D., Haith, A. M., Rossetti, Y., Shadmehr, R., and O'Shea, J. (2014). Contributions of the cerebellum and the motor cortex to acquisition and retention of motor memories. NeuroImage 98, 147-158. doi: 10.1016/j. neuroimage.2014.04.076

Hikosaka, O., Nakamura, K., Sakai, K., and Nakahara, H. (2002). Central mechanisms of motor skill learning. Curr. Opin. Neurobiol. 12, 217-222. doi: 10.1016/s0959-4388(02)00307-0

Imamizu, H., Miyauchi, S., Tamada, T., Sasaki, Y., Takino, R., Pütz, B., et al. (2000). Human cerebellar activity reflecting an acquired internal model of a new tool. Nature 403, 192-195. doi: 10.1038/35003194

Jalali, R., Miall, R. C., and Galea, J. M. (2017). No consistent effect of cerebellar transcranial direct current stimulation on visuomotor adaptation. J. Neurophysiol. 118, 655-665. doi: 10.1152/jn.00896.2016 
Jayaram, G., Galea, J. M., Bastian, A. J., and Celnik, P. (2011). Human locomotor adaptive learning is proportional to depression of cerebellar excitability. Cereb. Cortex 21, 1901-1909. doi: 10.1093/cercor/bhq263

Jayaram, G., Tang, B., Pallegadda, R., Vasudevan, E. V., Celnik, P., and Bastian, A. (2012). Modulating locomotor adaptation with cerebellar stimulation. J. Neurophysiol. 107, 2950-2957. doi: 10.1152/jn.00645.2011

Karni, A., Meyer, G., Jezzard, P., Adams, M. M., Turner, R., and Ungerleider, L. G. (1995). Functional MRI evidence for adult motor cortex plasticity during motor skill learning. Nature 377, 155-158. doi: 10.1038/377155a0

Kelly, R. M., and Strick, P. L. (2003). Cerebellar loops with motor cortex and prefrontal cortex of a nonhuman primate. J. Neurosci. 23, 8432-8444. doi: 10.1523/JNEUROSCI.23-23-08432.2003

Kumari, N., Taylor, D., and Signal, N. (2019). The effect of cerebellar transcranial direct current stimulation on motor learning: a systematic review of randomized controlled trials. Front. Hum. Neurosci. 13:328. doi: $10.3389 /$ fnhum. 2019.00328

Liew, S. L., Thompson, T., Ramirez, J., Butcher, P. A., Taylor, J. A., and Celnik, P. A. (2018). Variable neural contributions to explicit and implicit learning during visuomotor adaptation. Front. Neurosci. 12:610. doi: 10.3389/fnins.2018.00610

Manto, M., Gruol, D. L., Schmahmann, J. D., Koibuchi, N., and Rossi, F. (2013). Handbook of the Cerebellum and Cerebellar Disorders (pp. XXXIV, 2424). Netherlands: Springer.

Martin, T. A., Keating, J. G., Goodkin, H. P., Bastian, A. J., and Thach, W. T. (1996). Throwing while looking through prisms. I. Focal olivocerebellar lesions impair adaptation. Brain 119, 1183-1198. doi: 10.1093/brain/119.4.1183

Mathôt, S., Schreij, D., and Theeuwes, J. (2012). OpenSesame: an open-source, graphical experiment builder for the social sciences. Behav. Res. Methods 44, 314-324. doi: 10.3758/s13428-011-0168-7

Minarik, T., Berger, B., Althaus, L., Bader, V., Biebl, B., Brotzeller, F., et al. (2016). The importance of sample size for reproducibility of tDCS effects. Front. Hum. Neurosci. 10:453. doi: 10.3389/fnhum.2016.00453

Morton, S. M., and Bastian, A. J. (2006). Cerebellar contributions to locomotor adaptations during splitbelt treadmill walking. J. Neurosci. 26, 9107-9116. doi: 10.1523/JNEUROSCI.2622-06.2006

Motolese, A., Vignati, F., Brambilla, R., Cerati, M., and Passi, A. (2013). Interaction between a regenerative matrix and wound bed in nonhealing ulcers: results with 16 cases. Biomed. Res. Int. 2013:849321. doi: 10.1155/2013/849321

Moussa-Tooks, A. B., Burroughs, L. P., Rejimon, A. C., Cheng, H., and Hetrick, W. P. (2020). Cerebellar tDCS consistency and metabolite changes: a recommendation to decrease barriers to replicability. Brain Stimul. 13, 1521-1523. doi: 10.1016/j.brs.2020.08.005

Oldfield, R. C. (1971). The assessment and analysis of handedness: the Edinburgh inventory. Neuropsychologia 9, 97-113. doi: 10.1016/0028-3932(71) 90067-4

Panouillères, M. T., Joundi, R. A., Brittain, J.-S., and Jenkinson, N. (2015). Reversing motor adaptation deficits in the ageing brain using non-invasive stimulation. J. Physiol. 593, 3645-3655. doi: 10.1113/JP270484

Pascual-Leone, A., Grafman, J., Clark, K., Stewart, M., Massaquoi, S., Lou, J. S., et al. (1993). Procedural learning in Parkinson's disease and cerebellar degeneration. Ann. Neurol. 34, 594-602. doi: 10.1002/ana.410340414

Penhune, V. B., and Steele, C. J. (2012). Parallel contributions of cerebellar, striatal and M1 mechanisms to motor sequence learning. Behav. Brain Res. 226, 579-591. doi: 10.1016/j.bbr.2011.09.044

Poortvliet, P., Hsieh, B., Cresswell, A., Au, J., and Meinzer, M. (2018). Cerebellar transcranial direct current stimulation improves adaptive postural control. Clin. Neurophysiol. 129, 33-41. doi: 10.1016/j.clinph.2017.09.118

Priori, A., Ciocca, M., Parazzini, M., Vergari, M., and Ferrucci, R. (2014). Transcranial cerebellar direct current stimulation and transcutaneous spinal cord direct current stimulation as innovative tools for neuroscientists. J. Physiol. 592, 3345-3369. doi: 10.1113/jphysiol.2013.270280

Rahman, A., Toshev, P. K., and Bikson, M. (2014). Polarizing cerebellar neurons with transcranial direct current stimulation. Clin. Neurophysiol. 125, 435-438. doi: 10.1016/j.clinph.2013.10.003

Rasch, B., Gais, S., and Born, J. (2009). Impaired off-line consolidation of motor memories after combined blockade of cholinergic receptors during REM sleep-rich sleep. Neuropsychopharmacology 34, 1843-1853. doi: 10.1038/npp. 2009.6
Rauscher, M., Yavari, F., Batsikadze, G., Ludolph, N., Ilg, W., Nitsche, M. A., et al. (2020). Lack of cerebellar tDCS effects on learning of a complex whole body dynamic balance task in middle-aged (50-65 years) adults. Neurol. Res. Pract. 2:38. doi: 10.1186/s42466-020-00085-x

Reis, J., Schambra, H. M., Cohen, L. G., Buch, E. R., Fritsch, B., Zarahn, E., et al. (2009). Noninvasive cortical stimulation enhances motor skill acquisition over multiple days through an effect on consolidation. Proc. Natl. Acad. Sci. U S A 106, 1590-1595. doi: 10.1073/pnas.0805413106

Rezaee, Z., and Dutta, A. (2019). Cerebellar lobules optimal stimulation (CLOS): a computational pipeline to optimize cerebellar lobule-specific electric field distribution. Front. Neurosci. 13:266. doi: 10.3389/fnins.2019. 00266

Samaei, A., Ehsani, F., Zoghi, M., Hafez Yosephi, M., and Jaberzadeh, S. (2017). Online and offline effects of cerebellar transcranial direct current stimulation on motor learning in healthy older adults: a randomized doubleblind sham-controlled study. Eur. J. Neurosci. 45, 1177-1185. doi: 10.1111/ejn. 13559

Saucedo Marquez, C. M., Zhang, X., Swinnen, S. P., Meesen, R., and Wenderoth, N. (2013). Task-specific effect of transcranial direct current stimulation on motor learning. Front. Hum. Neurosci. 7:333. doi: 10.3389/fnhum.2013. 00333

Savic, B., Cazzoli, D., Müri, R., and Meier, B. (2017). No effects of transcranial DLPFC stimulation on implicit task sequence learning and consolidation. Sci. Rep. 7:9649. doi: 10.1038/s41598-017-10128-0

Schlerf, J. E., Galea, J. M., Spampinato, D., and Celnik, P. A. (2015). Laterality differences in cerebellar-motor cortex connectivity. Cereb. Cortex 25, 1827-1834. doi: 10.1093/cercor/bht422

Shah, B., Nguyen, T. T., and Madhavan, S. (2013). Polarity independent effects of cerebellar tDCS on short term ankle visuomotor learning. Brain Stimul. 6, 966-968. doi: 10.1016/j.brs.2013.04.008

Shimizu, R. E., Wu, A. D., Samra, J. K., and Knowlton, B. J. (2017). The impact of cerebellar transcranial direct current stimulation (tDCS) on learning fine-motor sequences. Philos. Trans. R. Soc. Lond. B Biol. Sci. 372:20160050. doi: $10.1098 /$ rstb.2016.0050

Shin, J. C., and Ivry, R. B. (2003). Spatial and temporal sequence learning in patients with Parkinson's disease or cerebellar lesions. J. Cogn. Neurosci. 15, 1232-1243. doi: 10.1162/089892903322598175

Sriraman, A., Oishi, T., and Madhavan, S. (2014). Timing-dependent priming effects of tDCS on ankle motor skill learning. Brain Res. 1581, 23-29. doi: 10.1016/j.brainres.2014.07.021

Stagg, C. J., Antal, A., and Nitsche, M. A. (2018). Physiology of transcranial direct current stimulation. J. ECT 34, 144-152. doi: 10.1097/YCT.0000000000000510

Stagg, C. J., Jayaram, G., Pastor, D., Kincses, Z. T., Matthews, P. M., and JohansenBerg, H. (2011). Polarity and timing-dependent effects of transcranial direct current stimulation in explicit motor learning. Neuropsychologia 49, 800-804. doi: 10.1016/j.neuropsychologia.2011.02.009

Stoodley, C. J., Valera, E. M., and Schmahmann, J. D. (2012). Functional topography of the cerebellum for motor and cognitive tasks: an fMRI study. NeuroImage 59, 1560-1570. doi: 10.1016/j.neuroimage.2011.08.065

Taubert, M., Stein, T., Kreutzberg, T., Stockinger, C., Hecker, L., Focke, A., et al. (2016). Remote effects of non-invasive cerebellar stimulation on error processing in motor re-learning. Brain Stimul. 9, 692-699. doi: 10.1016/j.brs. 2016.04.007

Thach, W. T. (1998). What is the role of the cerebellum in motor learning and cognition? Trends Cogn. Sci. 2, 331-337. doi: 10.1016/s1364-6613(98) 01223-6

Thair, H., Holloway, A. L., Newport, R., and Smith, A. D. (2017). Transcranial direct current stimulation (tDCS): a beginner's guide for design and implementation. Front. Neurosci. 11:641. doi: 10.3389/fnins.2017.00641

van Dun, K., Bodranghien, F. C., Marien, P., and Manto, M. U. (2016). tDCS of the cerebellum: where do we stand in 2016? Technical issues and critical review of the literature. Front. Hum. Neurosci. 10:199. doi: 10.3389/fnhum.2016. 00199

Walker, M. P., Brakefield, T., Morgan, A., Hobson, J. A., and Stickgold, R. (2002). Practice with sleep makes perfect: sleep-dependent motor skill learning. Neuron 35, 205-211. doi: 10.1016/s0896-6273(02)00746-8

Wessel, M. J., Zimerman, M., Timmermann, J. E., Heise, K. F., Gerloff, C., and Hummel, F. C. (2016). Enhancing consolidation of a new temporal motor 
skill by cerebellar noninvasive stimulation. Cereb. Cortex 26, 1660-1667. doi: 10.1093/cercor/bhu335

Wiestler, T., McGonigle, D. J., and Diedrichsen, J. (2011). Integration of sensory and motor representations of single fingers in the human cerebellum. J. Neurophysiol. 105, 3042-3053. doi: 10.1152/jn.00106.2011

Witt, S. T., Laird, A. R., and Meyerand, M. E. (2008). Functional neuroimaging correlates of finger-tapping task variations: an ALE meta-analysis. NeuroImage 42, 343-356. doi: 10.1016/j.neuroimage.2008.04.025

Wolpert, D. M., Diedrichsen, J., and Flanagan, J. R. (2011). Principles of sensorimotor learning. Nat. Rev. Neurosci. 12, 739-751. doi: 10.1038/ nrn3112
Conflict of Interest: The authors declare that the research was conducted in the absence of any commercial or financial relationships that could be construed as a potential conflict of interest.

Copyright (c) 2021 Nguemeni, Stiehl, Hiew and Zeller. This is an open-access article distributed under the terms of the Creative Commons Attribution License (CC BY). The use, distribution or reproduction in other forums is permitted, provided the original author(s) and the copyright owner(s) are credited and that the original publication in this journal is cited, in accordance with accepted academic practice. No use, distribution or reproduction is permitted which does not comply with these terms. 